\title{
Proportional Fairness Based Energy Efficient Routing in Wireless Sensor Network
}

\author{
Abolfazl Mehbodniya ${ }^{1}$, Surbhi Bhatia ${ }^{2}$, Arwa Mashat ${ }^{3}$, Mohanraj Elangovan ${ }^{4, *}$ and \\ Sudhakar Sengan ${ }^{5}$
}

\footnotetext{
${ }^{1}$ Department of Electronics and Communications Engineering, Kuwait College of Science and Technology, Kuwait ${ }^{2}$ Department of Information Systems, College of Computer Sciences and Information Technology, King Faisal University, Saudi Arabia ${ }^{3}$ Department of Information Systems, College of Computing and Information Technology, King Abdulaziz University, Rabigh, Saudi Arabia ${ }^{4}$ Department of Computer Science and Engineering, K. S. Rangasamy College of Technology, Tiruchengode, 637215, Tamil Nadu, India ${ }^{5}$ Department of Computer Science and Engineering, PSN College of Engineering and Technology, Tirunelveli, 627152, Tamil Nadu, India *Corresponding Author: Mohanraj Elangovan. Email: csemohanraj@gmail.com

Received: 05 July 2021; Accepted: 10 August 2021
}

\begin{abstract}
Wireless Sensor Network (WSN) is an independent device that comprises a discrete collection of Sensor Nodes (SN) to sense environmental positions, device monitoring, and collection of information. Due to limited energy resources available at $\mathrm{SN}$, the primary issue is to present an energy-efficient framework and conserve the energy while constructing a route path along with each sensor node. However, many energy-efficient techniques focused drastically on energy harvesting and reduced energy consumption but failed to support energy-efficient routing with minimal energy consumption in WSN. This paper presents an energy-efficient routing system called Energy-aware Proportional Fairness Multi-user Routing (EPFMR) framework in WSN. EPFMR is deployed in the WSN environment using the instance time. The request time sent for the route discovery is the foremost step designed in the EPFMR framework to reduce the energy consumption rate. The proportional fairness routing in WSN selects the best route path for the packet flow based on the relationship between the periods of requests between different SN. Route path discovered for packet flow also measure energy on multi-user route path using the Greedy Instance Fair Method (GIFM). The GIFM in EPFMR develops node dependent energy-efficient localized route path, improving the throughput. The energy-aware framework maximizes the throughput rate and performs experimental evaluation on factors such as energy consumption rate during routing, Throughput, RST, node density and average energy per packet in WSN. The Route Searching Time (RST) is reduced using the Boltzmann Distribution (BD), and as a result, the energy is minimized on multi-user WSN. Finally, GIFM applies an instance time difference-based route searching on WSN to attain an optimal energy minimization system. Experimental analysis shows that the EPFMR framework can reduce the RST by $23.47 \%$ and improve the throughput by $6.79 \%$ compared with the state-of-the-art works.
\end{abstract}

Keywords: Wireless sensor network; proportional fairness; multi-user routing; energy consumption; greedy instance fair; Boltzmann distribution

This work is licensed under a Creative Commons Attribution 4.0 International License, which permits unrestricted use, distribution, and reproduction in any medium, provided the original work is properly cited. 


\section{Introduction}

Efficient route identification is one of the most promising approaches in WSN. For example, if a sensor node wants to send data packets to the destination_node (DESTN_Node) through the sink, efficient route identification reduces the energy consumption of nodes and improves the network's lifetime. Many papers have been published to address the route identification problem. In [1,2], balancing energy during sensing and communication was performed based on the distributed algorithm to balance energy allocation efficiently. A Store Carry and Forward (SCF) mechanism was designed by applying the mathematical formulation to identify the shortest route to Base Station (BS). In [3], a method was presented to achieve high energy savings using optimization mechanisms that improved the battery lifetime with cooperative game theory. The cooperative theory applied in [4] has also been shown to consume less energy during packet forwarding than the non-cooperative principle. A quorum-based energy efficient protocol to minimize collision and improve transmission rate was presented in [5].

In recent years, improving the network lifetime by providing efficient routing has generated interest in WSN [6]. The methods involved harvesting energy using dedicated radio frequency and explored the possibility of increasing the network lifetime, resulting in energy fairness. This has motivated researchers to develop energy fairness mechanisms [7], resulting in an improved energy consumption rate but compromising transmission power. The ways to improve data transmission have been done through energy-optimized mechanisms [8], wherein transmission is performed based on open and closed-loop parameters. However, the delay factor for data transmission was not concentrated.

One of the most popular techniques in WSN for efficient routing is the design of an effective means for clustering. Cluster-based routing was introduced in [9] based on the cluster's energy density to reduce delay. A representative path was determined [10] by generating an algorithm for clusters to improve node lifetime. A trust aware routing protocol was designed in [11] using a Bayesian network. In [12], opportunistic routing was designed to improve the number of transmissions using link correlation. Data integrity [13] was measured using a multipath dynamic routing algorithm to reduce the delay involved during routing. In [14], a Secure Quality of Service Routing and communication sing Hierarchical Key Ranking in Mobile Ad hoc Network was presented. In [15], security groups have been formed, and the communication is done based on key mechanisms. In [16], Blockchain technology with its essential characteristics and applications are discussed. Particle Swarm Optimization algorithm [17] helps reduce SN's energy and prevent energy utilization by Low-energy adaptive clustering hierarchy protocol while routing the data sent to the destination or BS and results in data loss [18].

This paper develops an energy-efficient routing framework to support energy-efficient routing with minimal energy consumption in WSN. The following are the main contributions of this research: (a) A PFMR is developed with preference value to reduce the energy consumption during routing, and the results are supported by Network Simulation (NS-2); (b) the significance of considering interference rate in GIFM is demonstrated through simulation results with different node density; (c) an efficient design of BD considering the traversing time to reduce the RST between SN in WSN [19].

The remainder of this article is organized as follows. Section 2 presents the formulation of an energy-efficient routing framework called EPFMR in WSN and explains it with the help of the block diagram. Experimental results with parametric definitions are provided in Section 3. In Section 4, our proposal is compared to the other routing mechanisms. The paper concludes with a summary of our contributions in Section 5 .

\section{Energy-Aware Proportional Fairness Multi-User Routing Framework}

In this section, we first described the principles of the PFMR, then the GIFM to improve the instantaneous throughput rate. Finally, the construction of BD is made efficiently to reduce the RST considerably in WSN. Fig. 1 shows the block diagram of the EPFMR method [20]. 


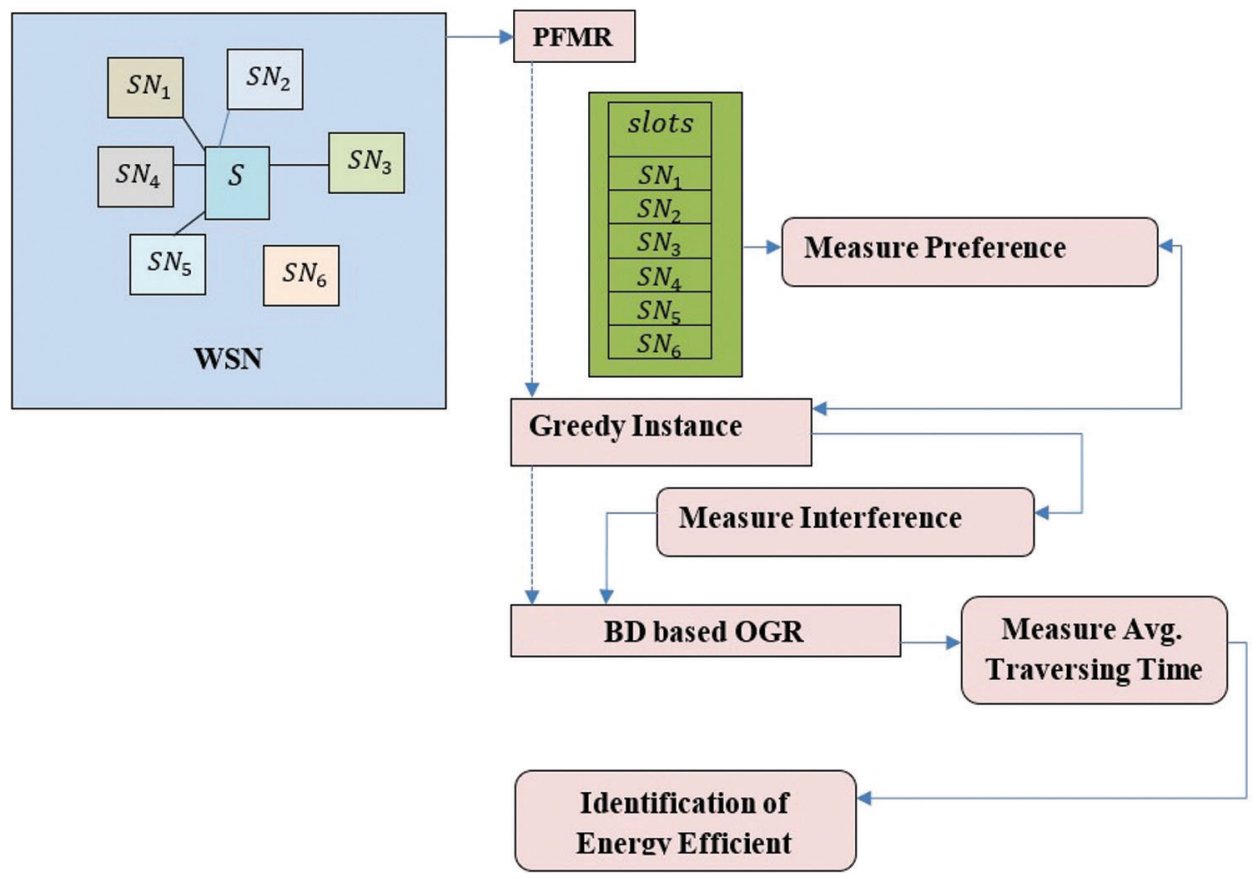

Figure 1: Proposed model of EPFMR

The EPFMR approach is divided into 3 sections to minimize the energy consumption levels during route discovery and improves throughput by minimizing interference during route discovery and RST [21]. PFMR is designed with slots for each $\mathrm{SN}$, and the next $\mathrm{SN}$ is assigned to a specific slot based on the preference value [22]. This process is repeated until all the SNs are assigned to a slot. Next, the interference rate is identified using the GIFM. Finally, according to the traffic (i.e., node density) and average traversing time, the Optimum Global Route (OGR) is measured, resulting in an energy-efficient route. A detailed description of each part is described in the forthcoming sections [23].

\subsection{Design of Proportional Fairness Multi-User Routing}

PFMR is presented in the conceptual methodology to minimize the energy consumption levels in WSN. The PFMR is deployed in WSN with the help of request time and serves as the first step in the EPFMR framework. Packet flow is performed in an efficient manner using the relationship between the periods of requests. Fig. 2 shows the design of PFMR using the design of the EPFMR framework in WSN [24].

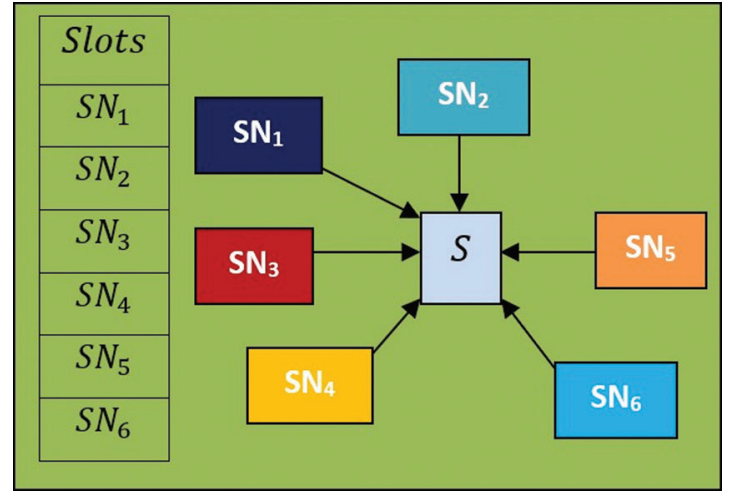

Figure 2: Design of PFMR in WSN 
Let us consider a scenario with ' $n$ ' $\mathrm{SN}$ (i.e., multi-users). ' $S N_{i}$ ' in the network is placed randomly within the sink ' $S$ ' section. Therefore, the EPFMR framework invokes multi-user as given below:

$S \rightarrow S N=\sum_{i=1}^{n} S N_{i}$

Eq. (1) assumes that ' $n$ ' $\mathrm{SN}$ requirements forward data packets from the sink node ' $S$ ' to ' $n$ ' DESTN_Node with varying transmission rates. Then, the EPFMR framework uses slots 'Slot $t_{i}$ ', through which the time is split into trivial gaps. With the help of 'Slots' in the EPFMR framework, only one SN transmits data packets. During the next slot assignment, in 'Slot $t_{i+1}$ ', the network evaluates the slot rates 'SR' by measuring Signal to Interference Plus Noise Ratio. The slot rates are obtained by deriving equilibrium between the prevailing slot rates and fairness [25].

The PFMR [26] in the proposed framework is performed by comparing the feasible slot rate for each SN to its average slot rate called the preference SN. The SN in WSN with a higher preference value is then selected for transmission during the next slot and is mathematically formulated as given below.

$P V_{S N_{i}}(n+1)=\frac{S N_{i}(n+1)}{A V G\left(S N_{i \rightarrow i+1}\right) * n}$

From Eq. (2), the preference value ' $P V$ ' is assigned for each $\mathrm{SN}$. ' $S N_{i}$ ' is obtained using the overall slot rate $S N_{i}(n+1)$ and average slot rate ' $A V G\left(S N_{i \rightarrow i+1}\right) * n$ '. The EPFMR framework selects the best route path for data packet transmission based on each $\mathrm{SN}$ request period. Therefore, by applying preference value-based slot data packet transmission, the EPFMR reduces the energy consumption during routing in WSN [27].

\subsection{Preference Value Multi-User Routing Algorithm}

Initialize: ' $S N_{i}={ } S N_{1}, S N_{2}, S N_{3} \ldots S N_{i}$ ' Sink, ' $S$ '

Output: Energy Efficient Routing

Step 1. Begin

Step 2. For Each $S N_{i}$ and Sink ' $S$ '

Step 3. Evaluates the SN ready for data packet transmission

Step 4. Evaluate preference value to decide upon the next SN ' $n$ ' the slot

Step 5. End For

Step 6. End

The preference value multi-user routing algorithm improves the energy consumption during routing in WSN. The algorithm involves two parts. The first part senses the SN that are ready for data packet transmission. Upon successful identification of SN, the preference value is evaluated for each SN to decide upon the sensor node to be selected as the node for packet transmission. With this, the energy consumption during routing is significantly reduced.

\subsection{Design of Greedy Instance Fair Method}

With the efficient route path discovery using the PFMR, GIFM is applied to the EPFMR framework to minimize interference during routing. The GIFM in EPFMR develops the SN dependent energy-efficient localized route path searching probability. Upon successful identification of route path discovery, GIFM is 
applied in the EPFMR framework. Each link with the SN and BS is allocated with an implicit demand according to the interference-free data packet. Next, the greedy instance is applied for each link between the SN and the BS. Then, each SN is assigned a slot. Based on the interference rate, the links are decreased. This process is performed until a fair route with reduced interference is evolved. As a result, the throughput rate of data packet transmission between $\mathrm{SN}$ is increased. Tab. 1 given below shows the throughput-improved GIFM using interference rate.

Table 1: GIFM using interference rate

\begin{tabular}{llll}
\hline Source (SRC)_node & DESTN_node & Hop node & Interference rate $(k b p s)$ \\
\hline $\mathrm{SN}_{1}$ & $\mathrm{SN}_{5}$ & $S N_{2}, S N_{3}, S N_{4}$ & 140 \\
$\mathrm{SN}_{1}$ & $\mathrm{SN}_{5}$ & $\mathrm{SN}_{3}, S N_{4}$, & 125 \\
$\mathrm{SN}_{1}$ & $\mathrm{SN}_{5}$ & $S N_{4}, S N_{5}, S N_{6}, S N_{7}$, & 110 \\
$\mathrm{SN}_{1}$ & $\mathrm{SN}_{5}$ & $S N_{3}, S N_{4}, S N_{5}$ & 105 \\
\hline
\end{tabular}

The analysis of a SRC Node ' $S N_{1}$ ' with the DESTN_Node ' $S N_{5}$ ', there are four routes (i.e., hop nodes) identified as ' $S N_{2}, S N_{3}, S N_{4}$ '; ' $S N_{3}, S N_{4}$ '; ' $S N_{4}, S N_{5}, S N_{6}, S N_{7}$ ' and ' $S N_{3}, S N_{4}, S N_{5}$ ' each having a different inference rate. By applying GIFM, the hop nodes, ' $S N_{3}, S N_{4}, S N_{5}$ ' with minimum interference rate of '105', is finally selected, thus improving the throughput rate.

\subsection{Construction of Boltzmann Distribution}

In this section, an OGR for SRC_DESTN pairs is designed using the BD to reduce the RST. The objective of the design of BD for multi-user routing in WSN is to update the request time based on the volume of the traffic. Based on the traversing time and volume of the traffic, BD for multi-user routing is designed in the EPFMR framework, as shown in Fig. 3. BD for multi-user routing involves an iterative ' $O$ ' value updating to search the route optimality and time optimality for a given source-destination pair. The ' $O$ Value' represented as ' $O(i, j)$ ', is the minimum traversing time to the DESTN_Node $D N_{d}$ with the assumption that a SN traverses from ' $i$ ' to ' $j$ '. The BD for multi-user routing with minimum RST is then formulated as given below:

$O\left(S N_{i}, D N_{d}\right) \rightarrow t\left(S N_{i}, D N_{d}\right)+\min O\left(S N_{i}, D N_{d}\right)$

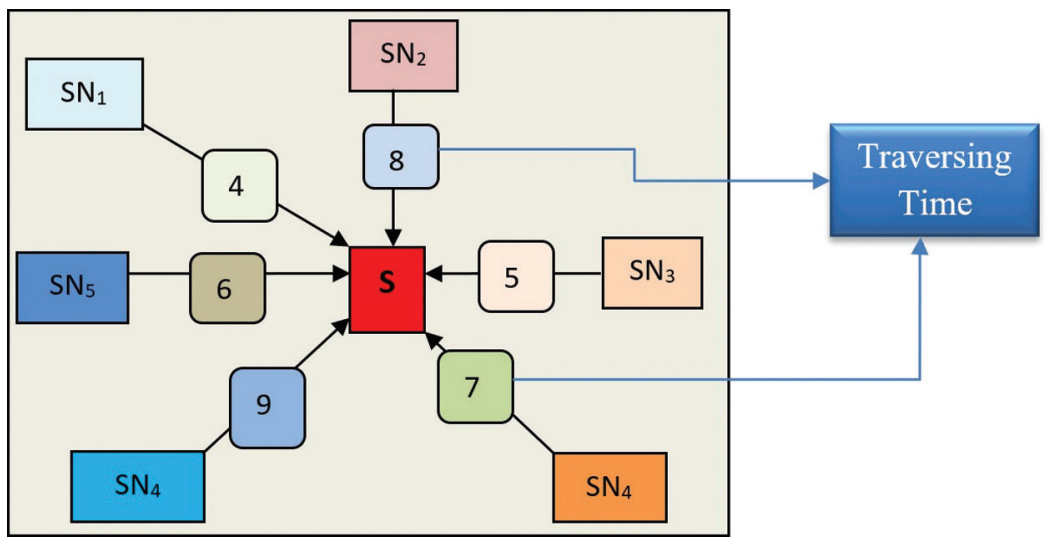

Figure 3: Optimum route search for S-D pairs 
Eq. (3), ' $t(i, j)$ ' refers to the traversing time from $S N_{i}$ to $D N_{d}$ for ' $d$ ' DESTN_Node. The basic idea of BD for multi-user routing is to measure the average traversing time for the source-destination pairs. The fundamental rule of the traffic assignment strategy is to calculate the average travelling time for the overall Origin-DESTN pairs using Eq. (3). GIFM and BD for multi-user routing follow an instance timebased route searching on WSN to reduce the RST.

The instance time-based route searching on WSN uses a threshold function ' $\alpha$ '. A significant value for ' $\alpha$ ' is assigned when the node density is high, whereas less value for ' $\alpha$ ' is assigned when the node density is low. Therefore, the value for ' $\alpha$ ' is set based on the node density. However, only a tradeoff is obtained. So, to reduce the RST, the EPFMR framework using BD for multi-user routing with delay factor is designed with the aid of a threshold function ' $\alpha$ ' and is formulated as given below.

$\alpha_{i}\left(S N_{d}\right)=\frac{O\left(S N_{i}, S N_{d}\right)}{N D}$

From Eq. (4), the threshold function ' $\alpha_{i}\left(S N_{d}\right)$ ' is derived by obtaining the ratio of multi-user routing with minimum RST ' $O\left(S N_{i}, D N_{d}\right)$ ' and the number of nodes in the network ' $N D$ '. As a result, the delay factor in identifying the OGR for SRC-DESTN pairs is obtained by reducing the RST.

\section{Experimental Settings}

In this section, we simulated the effectiveness of EPFMR's new framework. In WSN, the EPFMR model was evaluated by comparing it to Traditional Underwater acoustic sensor networks with Learning Automatacluster (TULA) and Store Carry and Forward (SCF). The simulation parameters used to execute the experimental studies are declared below in Tab. 2. The SN in the EPFMR system has a consistent topology.

Table 2: Specifications for simulation

\begin{tabular}{ll}
\hline Limits & Values \\
\hline Network simulation area & $1500 \mathrm{~m} \times 100 \mathrm{~m}$ \\
Number of SN & $10,20,30,40,50,60,70$ \\
Data packets & $8,16,24,32,40,48,56$ \\
Communication range & $30 \mathrm{~m}$ \\
Node speed & $0-10 \mathrm{~ms}$ \\
Test time & $1500 \mathrm{~s}$ \\
Test counts & 7 \\
\hline
\end{tabular}

The network is comprised of 70 nodes that are distributed randomly throughout the WSN and increases traffic every $10 \mathrm{~ms}$. The sink node receives network packets from various SN ranging from 8 to 56 and sends the data to the sink node. Each data packet size ranges from 100 to $512 \mathrm{~kb}$. The simulation time ranges from 500 to $1500 \mathrm{~ms}$, and the following performance measures are tested in WSN: power consumption during route discovery, average energy per packet, throughput, and RST.

The device of the energy consumed by a single SN and the total SN in a WSN is the energy consumed during route discovery.

$E C=$ Energy $_{S N} *$ Energy $_{S \rightarrow D N_{d}}$ 
In Eq. (5), ' $E C$ ' is the energy consumption during which 'Energy $\mathrm{SN}_{\mathrm{SN}}$ ' represents the energy consumed by each SN to reach sink node ' $S$ ' and 'Energy ${ }_{S \rightarrow D N_{d}}$ ' represents the energy consumed from the sink node ' $S$ ' to DESTN_Node ' $D N_{d}$ '.

Throughput is the ratio of packets sent by the SRC Node to the sink and packets appropriately collected by the respective DESTN Node via the sink node.

$T=\frac{D P_{S}}{D P_{D N_{d}}} * 100$

From Eq. (6), the throughput ' $T$ ' is measured using data packets sent by the SRC_Node. ' $D P_{s}$ ' and data packets are received at the destination end ' $D P_{d n d}$ '. Time taken for route search is the difference between the end time and start time for route search by the sink in WSN.

$R S_{t}=\left(\right.$ End time $_{R S}-$ Start time $\left.e_{R S}\right) * N D$

From Eq. (7), the time for route search is measured using ' $R S_{t}$ ', whereas 'End_Time $R S$ ' represents the end time for route search by the sink and 'Start_Time $R S$ ' represents the start time for route search by the sink in WSN. The average energy packet is measured using the total energy required for each packet to the packet size and are formulated below in Eq. (8).

$A E P P=\frac{\text { Total energy required }}{\text { Packet size }}$

\section{Result and Discussion}

In this section, the EPFMR's new framework results were evaluated and compared with two previous TULA and SCF techniques in WSN. The EPFMR framework's nodes are linked in a consistent topology. The following parameters, such as energy consumption during route discovery, throughput, RST, and energy consumed per packet in WSN, are evaluated to determine the effectiveness of the EPFMR protocol.

\subsection{Impact of Energy Consumption During Routing}

The values obtained from Eq. (5) are presented in Tab. 3 for various node densities using the presented EPFMR framework and extensively compared with TULA and SCF. Significant experimental findings were summarized and compared with the conventional TULA and SCF, respectively, to observe the efficiency of the EPFMR approach.

Table 3: Energy consumption during routing

\begin{tabular}{llll}
\hline \multirow{2}{*}{$\begin{array}{l}\text { Node } \\
\text { density }\end{array}$} & \multicolumn{3}{c}{ Energy consumption during routing $(\mathrm{J})$} \\
\cline { 2 - 4 } & EPFMR & TULA & SCF \\
\hline 10 & 35.32 & 56.12 & 61.35 \\
20 & 41.30 & 60.33 & 65.33 \\
30 & 44.53 & 69.56 & 74.56 \\
40 & 42.13 & 65.16 & 70.16 \\
50 & 47.21 & 67.24 & 72.24 \\
60 & 49.14 & 69.17 & 74.17 \\
70 & 45.22 & 50.25 & 55.25 \\
\hline
\end{tabular}


The results presented above Fig. 4 accept that as node density in WSN increases, so does energy usage during routing, which is significantly smaller while using EPFMR. So, the framework EPFMR is said to be highly energy-efficient compared with TULA and SCF. With node density 30 , the energy consumption during routing was $44.53 \mathrm{~J}$ using the EPFMR framework, whereas 69.56 and $74.56 J$ were consumed using TULA and SCF. With the use of PFMR, the energy consumption during route discovery is significantly decreased. To reduce the energy consumption during routing, Proportional Fairness assigns the SN in WSN with slots using preference value by comparing the feasible slot rate and average slot rate. As a result, the energy consumption during routing using the EPFMR framework is improved by $35.05 \%$ compared to TULA and $47.93 \%$ compared to SCF.

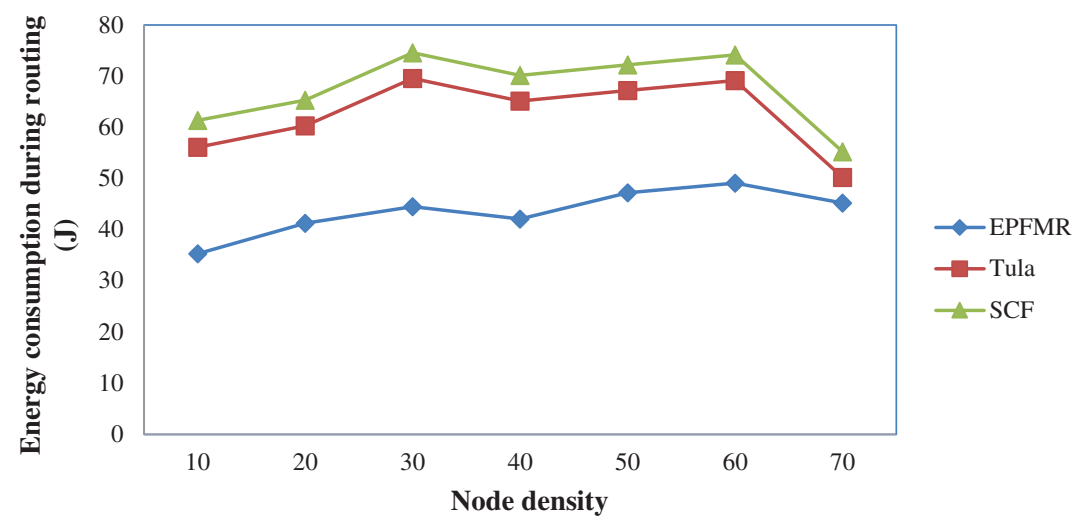

Figure 4: Measure of energy consumption

\subsection{Impact of Throughput}

Throughput for various data packets in the range of 8 to 56 is computed using Eq. (6). The number of SN in the testing method ranges from 10 to 70 . Tab. 4 shows the result of seven model testings used to determine the network throughput. The throughput for three different methods is expressed as a percentage. The throughput rates obtained in our approach are similar to those obtained with state-of-the-art techniques.

Table 4: Throughput analysis

\begin{tabular}{llll}
\hline \multirow{2}{*}{$\begin{array}{l}\text { Data } \\
\text { packets }\end{array}$} & \multicolumn{3}{c}{ Throughput (\%) } \\
\cline { 2 - 4 } & EPFMR & TULA & SCF \\
\hline 8 & 75.23 & 63.45 & 51.16 \\
16 & 69.13 & 60.11 & 50.08 \\
24 & 76.19 & 71.17 & 67.14 \\
32 & 78.24 & 73.22 & 69.19 \\
40 & 72.48 & 67.46 & 63.43 \\
48 & 80.23 & 75.21 & 71.18 \\
56 & 79.14 & 74.12 & 70.09 \\
\hline
\end{tabular}

A comparison was made between the targeting results of throughput rate using EPFMR framework with two advanced methods TULA and SCF, and Fig. 5 represents the visual comparison based on the pertinent 
data provided. The throughput rate for data packets is given, with each SN sending different data packets to the sink node.

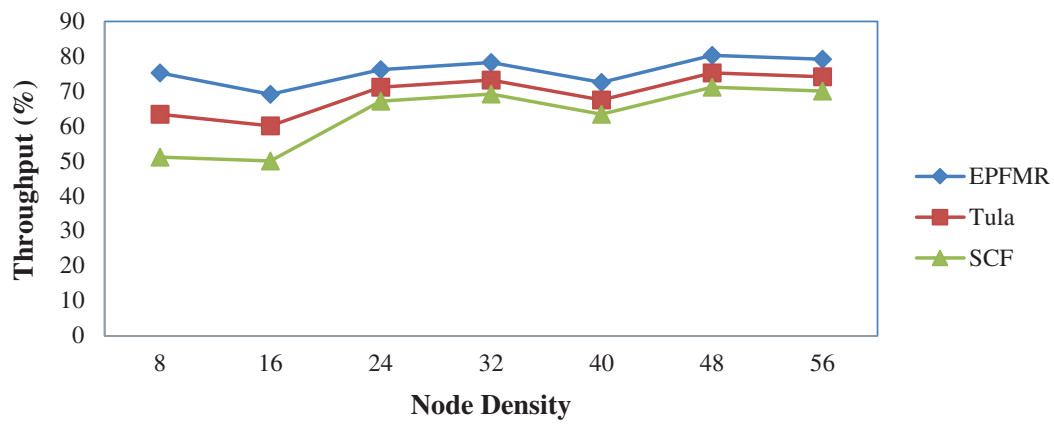

Figure 5: Measure of throughput

If the sink node receives 24 data packets, the throughput rate using the EPFMR framework will improve to $6.58 \%$ compared to TULA and $11.87 \%$ compared to SCF. This improvement is observed in the EPFMR framework by incorporating GIFM. The benefit of using GIFM in the EPFMR framework is that it performs data packet transmission based on the interference rate rather than directly. For effective transmission, the SN data packet with the lowest interference rate is chosen. Later, the data packet is transmitted between sensors and sink nodes using interference rate thus, increasing throughput by $10.95 \%$ vs TULA and $2.63 \% v s$. SCF.

\subsection{Impact of Route Searching Time}

Tab. 5 further compares the RST to node density at different time intervals in WSN. 70 SNs were used in the experimental studies, and the RST is measured in milliseconds $(m s)$.

Table 5: Route searching time

\begin{tabular}{llll}
\hline \multirow{2}{*}{$\begin{array}{l}\text { Node } \\
\text { density }\end{array}$} & \multicolumn{3}{c}{ Route searching time (ms) } \\
\cline { 2 - 4 } & EPFMR & TULA & SCF \\
\hline 10 & 1010 & 1255 & 1458 \\
20 & 1080 & 1330 & 1455 \\
30 & 1100 & 1350 & 1475 \\
40 & 1085 & 1330 & 1445 \\
50 & 1120 & 1370 & 1485 \\
60 & 1115 & 1365 & 1480 \\
70 & 1128 & 1378 & 1493 \\
\hline
\end{tabular}

Fig. 6 depicts the RST for the EPFMR framework, TULA, and SCF vs. 70 different SN. Compared to the other two methods, the RST returned by the EPFMR framework has increased slightly but not linearly with node density.

The RST for SRC-DESTN pairs using the EPFMR framework has been reduced. With the application of BD based on the instance time-based route searching, the traversing time and volume of the traffic are considered resulting in the minimized RST using the EPFMR framework. The BD with the aid of the ' $O$ ' 
value measures the average traversing time for the SRC_DESTN pairs, and therefore OGR is obtained for SRC_DESTN pairs. After identifying the OGR, the route is provided to the SRC_SN, reducing the RST by $15.82 \%$ and $31.12 \%$ compared to TULA and SCF, respectively.

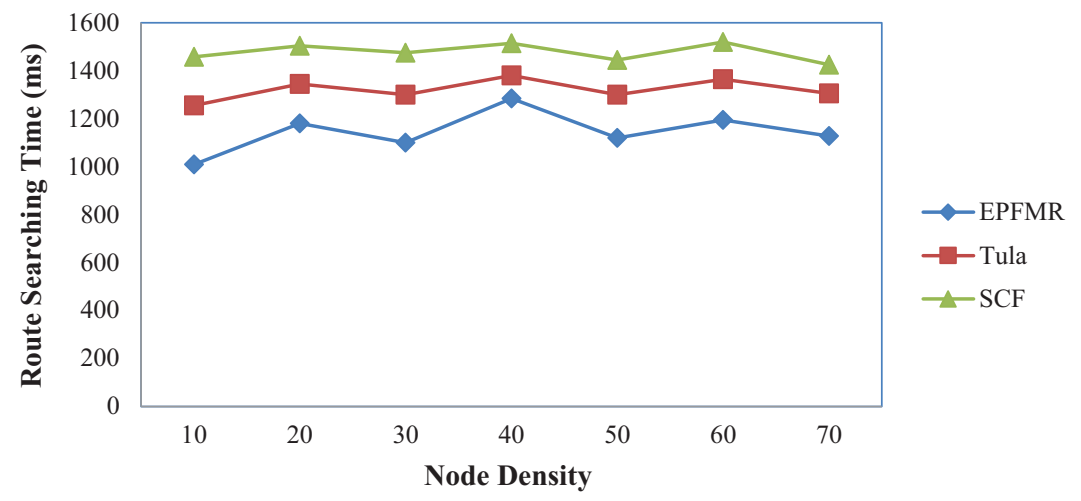

Figure 6: Measure of route searching time

\subsection{Impact of Average Energy Per Packet}

Eq. (8) is used to calculate the total energy per packet. Tab. 6 represents the average energy per packet for 56 data packets with node mobility of $15 \mathrm{~ms}$. Significant experimental results showed the comparison between the existing TULA and SCF frameworks to demonstrate the efficiency of the EPFMR framework.

Table 6: Average energy per packet

\begin{tabular}{llll}
\hline \multirow{2}{*}{$\begin{array}{l}\text { Data } \\
\text { packets }\end{array}$} & \multicolumn{3}{c}{ Average energy per packet (J) } \\
\cline { 2 - 4 } & EPFMR & TULA & SCF \\
\hline 8 & 4.25 & 5.25 & 6.35 \\
16 & 6.35 & 8.40 & 11.45 \\
24 & 10.14 & 12.19 & 15.24 \\
32 & 9.33 & 11.38 & 14.43 \\
40 & 14.59 & 16.64 & 19.69 \\
48 & 17.23 & 19.28 & 22.33 \\
56 & 15.21 & 17.26 & 20.29 \\
\hline
\end{tabular}

The effect of average energy per packet on varying data packets ranging from 8 to 56, and the average energy using three methods varies with data packet size. The results presented above confirm that because the source SN sends increased data packets to the sink node for route identification, so does the average energy per packet.

The average energy per packet for the three methods varies with the number of data packets sent. As displayed in Fig. 7, the EPFMR framework outperforms the other two different methods, TULA and SCF. This is due to the use of the threshold function ' $\alpha$ ' with $\mathrm{BD}$, which is determined based on node density. The multi-user route optimization ratio with the minimum RST is obtained, and the ratio of nodes is used for each packet sent by different nodes. This reduces the average energy per packet for route authentication using the EPFMR framework by $22.08 \%$ compared to TULA and $56.84 \%$ compared to SCF. 


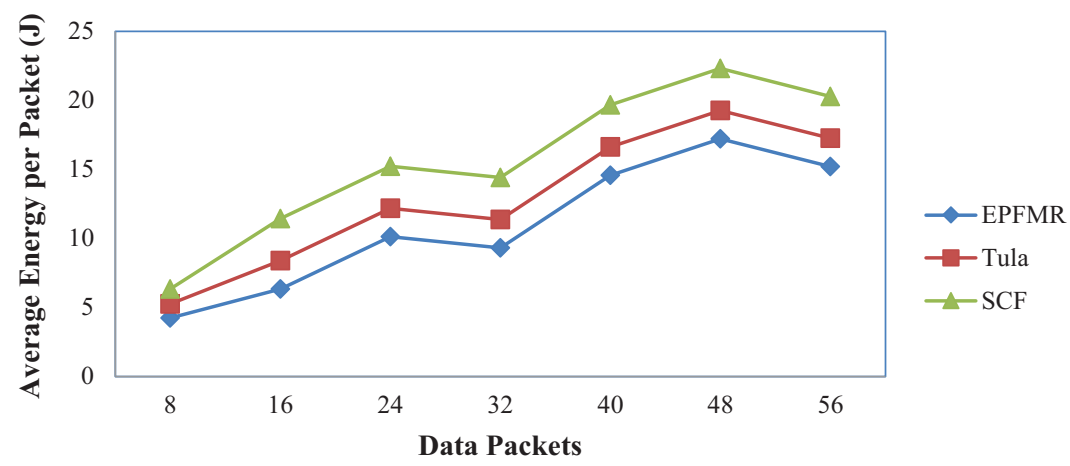

Figure 7: Measure of average energy per packet

\section{Conclusion}

This paper introduces a new EPFMR framework to reduce energy consumption during route discovery and RST with varying node density and packet size. This primary goal is met by identifying routes based on their periods and the percentage of interference observed by SN and transmitting network packets to the sink node accordingly. The goal implements a PFMR with BD, which saves energy and finding the optimal route for the various sensors with different packets in a WSN. As a result, throughput is enhanced, ensuring data packet transmission between the sender SRC and the DESTN Node via the sink node. An adaptation of the interference rate reduces the RST, ensuring the most negligible average energy per packet with the optimum solution. Experiments were performed out, and performance was compared with various process variables in terms of various performance measures, such as energy consumption during routing, throughput rate, average energy per packet, and RST. The findings demonstrate that the EPFMR framework outperforms TULA and SFC by optimizing energy consumption during routing by $41.49 \%$ and average energy per packet by $39.46 \%$.

Funding Statement: The authors received no specific funding for this study.

Conflicts of Interest: The authors declare that they have no conflicts of interest to report regarding the present study.

\section{References}

[1] J. Sorber, A. Balasubramanian, M. D. Corner, J. R. Ennen and C. Qualls, "Tula: Balancing energy for sensing and communication in a perpetual mobile system," IEEE Transactions on Mobile Computing, vol. 12, no. 4, pp. 804816, 2013.

[2] P. Kolios, V. Friderikos and K. Papadaki, "Energy-efficient relaying via store-carry and forward within the cell," IEEE Transactions on Mobile Computing, vol. 13, no. 1, pp. 202-215, 2014.

[3] C. Singhal, S. De, R. Trestian and G. Muntean, "Joint optimization of user-experience and energy-efficiency in wireless multimedia broadcast," IEEE Transactions on Mobile Computing, vol. 13, no. 7, pp. 1522-1535, 2014.

[4] N. Chen, M. Zhou, X. Dong, J. Qu, F. Gong et al., "Epidemiological and clinical characteristics of 99 cases of 2019 novel coronavirus pneumonia in Wuhan, China: A descriptive study," Lancet, vol. 395, no. 10223, pp. 507-513, 2020.

[5] S. Cacciapuoti, M. Caleffi, F. Tafuri, F. S. Cataliotti, S. Gherardini et al., "Quantum internet: Networking challenges in distributed quantum computing," IEEE Network, vol. 34, no. 1, pp. 137-143, 2020.

[6] P. Rajeswari and P. T. Kalaivaani, "Energy efficient routing protocol for wireless sensor networks using spatial correlation-based medium access control protocol compared with IEEE 802.11," in Int. Conf. on Process Automation, Control and Computing, Coimbatore, India, pp. 1-6, 2011. 
[7] S. Tanwar, S. Tyagi, N. Kumar and M. S. Obaidat, "LA-MHR: Learning automata-based multilevel heterogeneous routing for opportunistic shared spectrum access to enhance lifetime of WSN," IEEE Systems Journal, vol. 13, no. 1, pp. 313-323, 2019.

[8] T. Yang, T. Cui, C. Y. Xu, P. Ciais and P. Shi, "Development of a new IHA method for impact assessment of climate change on flow regime," Global and Planetary Change, vol. 156, no. 9, pp. 68-79, 2017.

[9] M. Faheem and V. C. Gungor, "Energy-efficient and QoS-aware routing protocol for wireless sensor networkbased smart grid applications in the context of industry 4.0," Applied Soft Computing, vol. 68, no. 7, pp. 910922, 2018.

[10] D. Mishra, S. De, S. Jana, S. Basagni, K. Chowdhury et al., "Smart RF energy harvesting communications: Challenges and opportunities," IEEE Communications Magazine, vol. 53, no. 4, pp. 70-78, 2015.

[11] S. Oluwole and M. V. Srivastava, "Features and futures of smart antennas for wireless communications: A technical review," Journal of Engineering Science and Technology Review, vol. 11, no. 4, pp. 8-24, 2018.

[12] M. M. Vegad, S. De and B. Lall, "Analysis of optimal carrier sensing range in wireless ad hoc networks with order-dependent capture capability," Wireless Personal Communications, vol. 81, no. 2, pp. 445-472, 2015.

[13] X. Wu, G. Chen and S. K. Das, "Avoiding energy holes in wireless sensor networks with nonuniform node distribution," IEEE Transactions on Parallel and Distributed Systems, vol. 19, no. 5, pp. 710-720, 2008.

[14] P. Loreti, A. Catini, M. De Luca, L. Bracciale, G. Gentile et al., "The design of an energy harvesting wireless sensor node for tracking pink iguanas," Sensors, vol. 19, no. 5, pp. 985, 2019.

[15] L. Song, C. Liu, H. Huang, H. Du and X. Jia, "Minimum connected dominating set under routing cost constraint in wireless sensor networks with different transmission ranges," IEEE Transaction on Network, vol. 27, no. 2, pp. 546-559, 2019.

[16] S. Adu-Manu, N. Adam, C. Tapparello, H. Ayatollahi and W. Heinzelman, "Energy-harvesting wireless sensor networks (EH-WSNs): A review," ACM Transaction on Sensor Network, vol. 14, no. 2, pp. 1-50, 2018.

[17] H. Jawad, R. Nordin, S. Gharghan, A. Jawad and M. Ismail, "Energy-efficient wireless sensor networks for precision agriculture: A review," Sensors, vol. 17, no. 8, pp. 1781, 2017.

[18] S. Nanthini, S. N. Kalyani and S. Sudhakar, "Energy efficient clustering protocol to enhance network lifetime in wireless sensor networks," Computers, Materials \& Continua, vol. 68, no. 3, pp. 3595-3614, 2021.

[19] A. Gumusalan, R. Simon and H. Aydin, "Dynamic modulation scaling enabled multi-hop topology control for time-critical wireless sensor networks," Wireless Network, vol. 26, no. 2, pp. 1203-1226, 2020.

[20] L. Song, K. Chai, Y. Chen, J. Loo, S. Jimaa et al., "Energy-efficient cooperative coalition selection in clusterbased capillary networks for CMIMO IoT systems," Computer Network, vol. 153, no. 3, pp. 92-102, 2019.

[21] S. Sudhakar and S. Chenthur Pandian, "Hybrid cluster-based geographical routing protocol to mitigate malicious nodes in mobile ad hoc network," International Journal of Ad Hoc and Ubiquitous Computing, vol. 21, no. 4, pp. 224-236, 2016.

[22] S. Sudhakar and S. Chenthur Pandian, "Investigation of attribute aided data aggregation over dynamic routing in wireless sensor," Journal of Engineering Science and Technology, vol. 10, no. 11, pp. 1465-1476, 2015.

[23] C. Sathiya Kumar, V. Priya, V. P. Sriram, K. Sankar Ganesh and G. Murugan, "An efficient algorithm for quantum key distribution with secure communication," Journal of Engineering Science and Technology, vol. 15, no. 1, pp. 77-93, 2020.

[24] N. Satheesh, D. Sudha, D. Suganthi, S. Sudhakar, S. Dhanaraj et al., "Certain improvements to location aided packet marking and DDoS attacks in internet," Journal of Engineering Science and Technology, vol. 15, no. 1, pp. $94-107,2020$.

[25] E. Mohanraj, K. Duraiswamy and K. Prasanth, "Secure QoS-based routing using hierarchical key ranking model in MANET," Journal of Circuits, Systems, and Computers, vol. 23, no. 9, pp. 1-15, 2014.

[26] E. Mohanraj and K. Duraiswamy, "Routing based data security in mobile ad hoc network using group key management," Journal of Computer Science, vol. 8, no. 10, pp. 1711-1717, 2012.

[27] A. Pushpalatha, R. Mahima, K. S. KiruthikRuba, E. Mohanraj, P. Rajaram et al., "Optimized data routing using PSO in WSN," International Journal of Advanced Science and Technology, vol. 29, no. 3, pp. 8298-8303, 2020. 